\title{
Learning to Die: Analysis of The Cultural Role of Voluntary Death
}

\author{
Niccolò Martini \\ University of Bologna, Italy
}

\begin{tabular}{|c|c|}
\hline ARTICLE INFO & ABSTRACT \\
\hline $\begin{array}{l}\text { Keywords: } \\
\text { Culture } \\
\text { End-of-Life } \\
\text { Euthanasia } \\
\text { Legalization } \\
\text { Voluntary }\end{array}$ & $\begin{array}{l}\text { Voluntary death is a morally and legally grey area in many countries } \\
\text { around the world. In my research I studied the topic of euthanasia and } \\
\text { assisted suicide in Italy. Specifically, I analyzed the relationship } \\
\text { between collective law and individual morality using as case study the } \\
\text { phenomenon of voluntary death, which has been making people talk } \\
\text { about itself in recent years precisely because of its as yet undefined } \\
\text { nature. Using a qualitative approach i.e., semi-structured interview, I } \\
\text { listened the voices of a representative sample of Italian doctors in order } \\
\text { to collect the opinion of the medical class i.e., the social group that } \\
\text { would be most affected by the possible legalization of euthanasia. It has } \\
\text { emerged, among other things, that Italy lacks a real education to death. } \\
\text { The research has opened a reflection on the range of voluntary death } \\
\text { within a Nation where it is illegal. Numerous studies have determined } \\
\text { the enormous symbolic baggage present within the concept of death, but } \\
\text { in the study of the legalization of voluntary death a new factor has } \\
\text { emerged: a legalization is not desired until the population receives a real } \\
\text { education on the idea of having to die. Like sex, death is still a taboo in } \\
\text { many societies around the world. Is it therefore necessary to fulfill a } \\
\text { death education before even start to talk about creating a general law. } \\
\text { This research has exalted not only a cultural deficiency but also the } \\
\text { desire to remedy it through education, in order to exorcise the fear of an } \\
\text { event that sooner or later everyone has to face. }\end{array}$ \\
\hline
\end{tabular}

\section{Introduction}

Social scientists have always tended to be particularly attracted to the phenomena related to death, since it is precisely in these liminal moments, between life and its cessation, that emerge many of the conceptions and beliefs that characterize a society.

The recent development of the Social Health Sciences has brought with it a new interest in the dying itself, thanks also to the innovations in the medical field that have allowed more and more people to survive for a relatively long period of time with a terminal disease.

In the last two decades, new practices have been introduced within the social-biological panorama, thanks to which an individual, under certain conditions, can voluntarily request that his life be ended through a rapid and painless injection. I'm talking about euthanasia and assisted suicide.

It is an unprecedented event that revolutionizes both the medical field and the society in which this practice is legalized. For the first time in the age of modern medicine, is allowed the possibility of dying rather than continuing with treatments.

Death becomes the ultimate cure.

* Corresponding author E-mail address: niccolo.martini2@gmail.com 
The introduction of such a practice completely redefines the medical ethics and the collective morality of society: the doctor must go against the Hippocratic oath and give death instead of fighting it, society must normalize suicide, usually criminalized.

In the following pages I'll expose the research about the end-of-life in Italy that I conducted for my master thesis. My intention has been to go beyond the simple understanding of how much the practice of euthanasia and assisted suicide is accepted in Italy (a country where such actions are still illegal), the main focus has been to problematize such events in order to understand what future developments there could be. Through a qualitative research methodology and a semi-structured interview, I collected mainly the voices of the medical class as it would be the social group that will be most affected by the possible legalization of euthanasia and assisted suicide.

Creating a collective norm to regulate a phenomenon as individual as death, puts in place the mechanics that not only touch the legal-institutional sphere but also the individual and cultural one. What correlation exists between collective law and individual morality? What problems must be resolved before proceeding with the creation of the norm? What situations are faced by doctors in the current condition of total illegality of voluntary death (in Italy)?

\section{Materials and Method}

The method adopted for this study is that of qualitative research. A total of 20 semi-structured interviews were conducted with as many physicians of various specialties. Since this is a very sensitive issue, it was decided to opt for this approach in order to bring out as much information as possible without excluding anything. The semi-structured interview was chosen because it was considered the best way to research this topic: the structured interview would have been too closed and limiting while the unstructured one would have risked causing an excessive digression and therefore going off topic.

Since this topic is not only morally delicate but also practically illegal, I decided to proceed using a "deductive" approach, i.e., starting from general cases and then narrowing the circle and going more and more into the details of the interviewee's thought. In this way I wanted to "break the ice" using a very famous case in Italy (Fabiano Antoniani and Marco Cappato) in order to bring out the opinions about it and then go more personal in a second moment. Through this method I wanted to prevent a premature closure of the interviewee, which tends to happen if, investigating the issues of the end of life, you immediately start the conversation trying to get the personal opinions of the subject.

The purpose of the interview was not only to bring out the opinions of the interviewee but also to create an opportunity for shared reflection. As much as a physician tends to reflect often on liminal situations between life and death, he or she is unlikely to use a socio-anthropological approach to voluntary end-of-life care. My desire was precisely to reflect together with them on these issues, in this way very often the doctors came to tell me somethings that they themselves had never thought of.

The physicians interviewed were selected on a voluntary basis, initially by asking various hospital companies and then the physicians themselves. Having initially encountered many problems in finding the subjects through health institutions and hospitals (reasoning as companies, they have proved much more reluctant to expose employees on a topic so legally sensitive), I proceeded to contact the regional Medical Order directly to ask them if it was possible to circulate my project among all members of the register. After doing so, the individual doctors interested to my research contacted me to organize an appointment. The group chosen in this way consists of a representative sample collected in a region of central- 
northern Italy, whose opinions allow to hypothesize what is the widespread thinking of the medical class.

In most cases (16 of 20), interviews took place at the physicians' workplaces (hospitals or private clinics); in a small percentage ( 2 of 20), interviews took place electronically (Skype) because physicians were abroad on business; in the remaining cases ( 2 of 20), they took place in the physicians' private homes (at their request).

Field research took place between December 2019 and February 2020. For privacy reasons it is not possible to say the name of the doctors, so I will refer to them with random alphabetical letters.

All the interviews have been transcribed in their entirety, obviously the original language is Italian but here they have been translated into English to facilitate understanding.

\section{Results and Discussion}

Famous is the case of Fabiano Antoniani who was blind and quadriplegic following a car accident on June 13,2014. Not wanting to remain in that condition, he begins to insist on asking to the Italian government the permission to end his life. This request has caused great uproar: the government did not know how to behave, and the Italian public opinion was split into two parts, those who supported the request of Antoniani and those who considered it morally unacceptable.

After years of requests, he decided to take the initiative and with the help of Marco Cappato went to Switzerland where he asked and gets permission to proceed with assisted suicide. By only partially moving the fingers of his hand, he was given a button to release the lethal dose of the drug into his IV.

On 27 February 2017 Fabiano Antoniani literally died by his own hand.

In Italy there is no law that explicitly denies any euthanasia/assisted suicide attempt, in fact those actions are never mentioned by name, but indirectly they are forbidden.

Paragraph 1 of Article 579 of the Italian Criminal Code states that "Whoever causes the death of a man with his consent shall be punished by imprisonment from six to fifteen years".

It is superfluous to note that although euthanasia is not mentioned explicitly, it is denied in its assumptions because it is forbidden to one person to cause the death of another even if the latter proves to be consenting. This is therefore a clear attack on any form of voluntary death, no matter whether there is consent or not, causing death is illegal.

However, what can be reflected upon most is the third point of the article, which states:

\section{The provisions relating to murder apply if the act is committed: \\ 1) against a person under the age of eighteen; \\ 2) against a person who is mentally ill, or who is in a state of mental deficiency, for another illness or for the abuse of alcohol or drugs; \\ 3) against a person whose consent has been obtained by the perpetrator by violence, threat or suggestion, or by deception.}

With these few lines, the article of the penal code sketches an important demarcation: in the cases of specific persons (who do not have the legal right to understand and want) the act of causing their death is punished using the rules governing the murder.

Saying this, the article argues something seemingly obvious, namely that in cases where there is a lack of voluntariness of the subject to whom the death is given, it necessarily speaks of 
murder. The interesting thing is what is implicitly said between the lines: cases other than those listed are not homicide. What are they? With what attribution are they punished?

This answer can already be found in the title of the next article, 580, called "instigation or help to suicide". The Italian legislative apparatus uses the word suicide to identify the voluntary death of a person, bringing with it all the negative meanings that historically and culturally this term possesses.

Being therefore illegal, once back from Switzerland Cappato decided to denounce himself in order to bring visibility to a theme too often left in the shadows. The trial lasted for years and ended only a few days before Christmas 2019 with the release of the indicted. His intent was to stir up the souls of the government in order to obtain a law, and even if this did not happen, the Italian Constitutional Court stated that under certain circumstances it is not punishable who helps another person to obtain death. The declaration has no legal value but constitutes a precedent, a first step.

This recent event highlights not only the current problems that revolve around the voluntary death but also highlights the need to study this topic at this moment in history.

Medical science of the last decades has made enormous technical progress so as to create new conditions of existence unimaginable before, such as PVS (Persistent Vegetative State). At the same time the practice has moved new ethical issues: with the possibility of performing heart transplants (which can only be performed using working hearts until the exact moment of explantation) has become necessary to redefine the concept of death in order to be able to explant the heart from patients in PVS without having to declare to have killed them. For this reason, in the '60s was born the concept of brain death (Singer, 1995). Biomedicine did not discover death but gained the structural power to be able to attest and define it.

New discoveries have created increasingly liminal situations in which a body can be kept alive mechanically through the aid of external devices creating gray situations of life/non-life. Despite this, death (and even more so voluntary death) remains an ideologically dense issue and therefore, from a cultural and institutional point of view, it becomes more difficult to regulate the new extreme situations that have been created.

The Oviedo Convention signed in 1997 was an important first international step towards a general regulation of bioethics. The intent of the treaty was to cross-guarantee human dignity in the face of constant progress in biomedicine. Wanting to constitute a framework (Andorno, 2005 ) to be implemented with subsequent protocols, it did not go into detail on the subject of the end-of-life and to date the three protocols that have been added concern human cloning (1998), organ transplantation (2002) and biomedical research (2004).

The most recent Guide on the decision-making process regarding medical treatment in end-oflife situations produced by the Council of Europe in 2014 focuses more on the topic of end-oflife showing the great interest that institutions have in wanting to manage these practices. Despite this, the document remains a guide without legal value: starting from the 4 general principles of bioethics (Beauchamp, Childress, 1979) outlines what are the subjects involved and what decision-making process to adopt in end-of-life situations, then it is up to individual doctors to decide whether to take a cue or not.

In Italy articles 2, 13 and 32 of the Constitution protect freedom of choice and human dignity, while the (partially decriminalized) article 580 of the Criminal Code already mentioned prohibits any form of aid and incitement to suicide. Supported by the Constitution, a few years ago was enacted the law that allows the execution of the D.A.T. (Disposizioni Anticipate di Trattamento - Anticipated Treatment Dispositions), also known as "biological will", thanks to which it is possible to fill out a document in which you certify that you want to renounce to 
medical treatment in the event of a serious health condition. I will speak later about their effectiveness within the Italian population.

Euthanasia is a theme that has entered the center of analysis in extremely recent times. Its removal from the scientific scene is due not only to the late emergence of medical anthropology and social health sciences (disciplines that prefer this type of analysis), but also to the constant cancellation of death from the intellectual landscape. The cessation of life is a powerful taboo, and as such is often ignored.

In the '60s Herman Feifel was among the first to analyze with a multidisciplinary method the concept of death through a collection of essays that used different perspectives (Feifel, 1965), in such a way as to bring out of the shadows a theme that deserved reflection.

Later, more and more studies on the conception of death in various social contexts and its denial came out (Becker, 1973), so when the advances in biomedicine allowed the emergence of the euthanasia phenomenon, the first scientific analyses about it began to emerge (Clark, 2002; Glover, 1990; Hayry, 1997; Jones et al., 2017; Kuuppelomaki, 2000).

Over time, more and more liminal cases have arisen that have confronted physicians with new bioethical situations, despite this, the actual legalization of euthanasia has occurred in very few states [Netherlands (2002); Belgium (2002); Luxemburg (2009); Colombia (2014); Canada (2016); South Korea (2018); Australia (only the state of Victoria, 2019); Spain (2021)]. The Spanish case is emblematic, which proceeded with legalization during the period when global health is focused on the Sars-CoV 2 pandemic.

The international scientific literature has produced over the years studies and analysis on euthanasia (Lewins, 1998; McDougall et al, 2008; Oduncu, 2003; Owen et al, 1992; Rosenfeld, 2004; Yolanda, 2018), as well as some analysis on the conception of death itself (Seale, 2000; Veena et al, 2016) but, unfortunately, the material concerning the Italian case is very scarce. The reasons for this absence can be traced in multiple factors: the presence of the Vatican State, the still high presence of the taboo of death, the constant cuts in research and health and a political class that rarely wants to deal with issues of this magnitude in parliament.

The result of this situation is a complete ostracism, the topic is discussed only when it is given great importance in the media following particular cases (Elena Moroni; Piergiorgio Welby; Eluana Englaro; Fabiano Antoniani to name a few), in which it is the individual (or his or her family) who must take the initiative to ask the government to act.

In the absence of a national law, each case must find its own specific way of resolution, and if explicit requests to Parliament have no effect, it is individuals, be they patients, family members or doctors, who must act, either by going against the law or by going to another country to obtain a legal solution.

For this reason, it is necessary to study what to date has no universal resolution but possesses infinite practical and moral nuances caused by its inherent liminality.

As I already mentioned, my research focused on the study of voluntary death in Italy using a qualitative analysis (interviews) of the social group that would be most affected in the event of a possible legalization of the phenomenon: doctors. For reasons of privacy, it is not possible to reveal the names of those who participated or the organizations to which they were affiliated, here I will list the results that emerged, what problems were raised by the doctors and what dynamics should be taken into account when talking about "legalizing euthanasia".

Anthropology is the social science that study humankind both as producer and as product of symbols,

medical anthropology started as a scientific field that wanted to study how the culture influence the medical professions, not only "other" medicines but our biomedicine as well (Eisenberg, 1997; Kleinman, Eisenberg, Good, 1978; Kleinman, 1980). This element is more exalted 
during an interview that want to examine one of the most symbolic moment of all the human life: death.

One of the first things to emerge (and one of the most important) concerns the influence that culture exerts not only on death itself (a subject already widely demonstrated by numerous studies) but also on the status/role of the social subject. In the case of the end-of-life this influence is reflected in the judgement of the doctors on the voluntary death: if at the beginning of the interview the opinion "pro euthanasia/assisted suicide" tended to emerge immediately because it was considered obvious that every human being could decide for himself, later on this position tended to be partially or totally retracted when the interviewee started to think about it "as a doctor and not as a citizen".

I deliberately structured the interview starting from a specific and well-known case (the already mentioned Fabiano Antoniani and Marco Cappato) and then gradually widening my view on the general legislation. In doing so, the first comments regarding the issue of the end of life concerned the need for everyone to be able to choose what to do with their own existence, however, as soon as the professional opinion took over the conversation, the initial ethical absolutism disappeared to leave the field to a greater level of problematization.

The main point that emerged during the interviews is that Death is still taboo, just as sex, and as a result the topic is taken out of the mind, ignored, with the consequent inability to deal with it the moment it comes to the door. In practice what the doctors have accused is the difficulty of creating a law on death in a society in which is ignored.

It has emerged that the discourse is not simply about the individual's desire to want a law, but rather about the actual effectiveness that the law can have in one specific context.

Law is not only an organizational tool but also a cultural product. In this regard, another particularly interesting element that emerged is the phenomenon that I called "reverse biomedicalisation". In Social Health Sciences the term biomedicalization (Clarke et al., 2003) indicates a tendency to see as biomedical phenomena those that are not necessarily biomedical, using a reductionist (everything can be explained through the biomedical lens) and exclusivist (what cannot be explained through the biomedical lens is not a health problem) perspective. By the term "reverse biomedicalisation" I mean the fact that in this case the phenomenon of biomedicalisation is not carried out by the medical class; on the contrary, it is the latter that fears that the population itself is doing so. In practice, I have been told many times how many patients are already asking for drugs driven by the desire to get everything immediately and thus solve a problem as quickly as possible.

In the case of the possible law on the end-of-life, the fear expressed to me concerns the fear of a possible reification of life itself, i.e., the fear that legalizing the voluntary end of life could push some people to use this possibility too lightly. Basically, it is feared that people use the biomedical lens too much to see life itself as a product to be disposed of when it is no longer convenient to preserve it.

It is interesting to note that in this case it is the medical class itself, often accused of reifying the body too much and seeing it as a machine to be adjusted, that criticizes the use of this attitude by the population.

This fact adds an extra element and shows further how much talking about the end of life implies bringing complex cultural dynamics into play.

However, the "cultural criticism" emerged against the medical class itself as well. The unpreparedness in the field of death concerns not only the population but also the medical class. The totality of cases has in fact expressed how much the same medical preparation is currently lacking on the subject of death. There is a widespread tendency to want more attention to bioethical issues, which are too often ignored both at university and at the school of 
specialization. Younger doctors with more recent testimonies about the current educational process in the medical faculty have highlighted the existence of a bioethics class during university, however, often goes unnoticed and doesn't teach what concern dealing with death. For example, H. told me:

H: Well, beat it more, don't do the course...I'll tell you again, for example, I took the bioethics course, it was a course like that, a few credits, a few hours, who cares, easy exam...instead, beat it more on these topics, organize seminars...on practical cases, in short, don't do the usual little lecture like that, where you'll be told two little...nonsense shit, and then...

All this implies that each doctor must train himself or herself in the end-of-life approach, increasing the possibility that situations may arise in which both the patient and the treating doctor have personal and interpersonal difficulties.

A: The problem, in my opinion, is that there is no medical training that would allow you to get to the suspension of treatment in a healthy, reasonable, shared way, but it could be done.

The real victim of this situation is the doctor-patient relationship, somewhat limited by the obscure presence of an issue that is difficult to manage on both sides. This event confirms the tendency of isolating the dying person analyzed by another social scientist (Bauman, 1995; Elias, 2011)

Not only the preparation is insufficient, it also more focus on the behavior of the professional rather than the ethical situation in which he/she can be involved:

$\mathrm{C}:[\ldots]$ ethics was already there in my day when these things were being done... ethics was approached in a different way, ethics was just as you put yourself as a professional, not ethics referring to these choices.

The element of cultural and didactic unpreparedness on the theme of death is one of the fundamental points that emerged from this study. In essence, it means that before reflecting on the creation and implementation of a law on death, one should work on the cultural basis on which such a law would be based.

The concept of voluntariness applied to death brings into play new factors and different ideologies, however, it has emerged that we should before work from the educational point of view on the concept of death per se.

Many physicians have used as an example to validate this idea the Italian situation regarding D.A.T. (Disposizioni Anticipate di Trattamento) commonly known as bilological wills (testamento biologico). This law allows individuals to create a document through which they can certify that they wish to renounce medical treatment (knowing that this will lead to their death) if they find themselves in serious and irreversible health conditions. Doctors have repeatedly pointed out to me that this law was approved in December 2017 and came into force in January 2018, but although it was strongly desired by a slice of the population, in recent years less than $1 \%$ have taken advantage of the possibility of drawing up a living will:

I: [...] you have seen the DAT, the data from the ministry tell us that the 0.01 ...in short, 60 thousand DAT deposited out of the 50 million people who can deposit them...after 
two years it means that it is not something that interests so much people...as a principle it interests many...

This is again a discrepancy between moral ideology and concrete practice when death, health and illness are involved, and this thing hasn't gone unnoticed by doctors either

What is the explanation behind these numbers? For example, $\mathrm{A}$ is convinced that this numbers are the symptom that this type of issue is niche and is only dealt with by the most educated part of the population:

A: for us who do this work is a continuous reflection that you do with yourself, with your colleagues always, every day in the end because ... death is here every day with us, the problem is that you never talk about it, you talk about it when it happens but people do not ... few people reflect on what is your future ... and they are still laws for a few ... they are laws that ... even the D.A.T. who makes them? They are made by the people who study...but not all of them, in my opinion, it is not something that can reach everyone, and it is wrong, because...it is a bit of a niche.

This is reflected in the already mentioned fear of the law that several doctors expressed. Such fear is moved by the idea that the population is not ready, and people can start to use euthanasia/assisted suicide in the same way as other medicines.

Despite this practical fear, another opinion that emerged regarding the actual situation of the medical system is the excessive legal pressure under which every physician is placed. Today every doctor is a fragile subject on a legal point of view because is everyday easier to accuse them of something and this, sometimes, push them to do defensive medicine just to avoid any jurisdictional implications.

This opens the reflection about the dynamics of Power between the State and the medical expertise. Obviously it is necessary a system of rules in order to avoid that physicians start to act as they prefer, but on the other hand when the rules are too strict, the physician can be put into the situation in which has to act in a way that is not convenient for the patient just to avoid any legal implication. The worst aspect of this dynamics is that the person that at the end suffer is always the patient, nor the physician or the politician.

It is interesting to note how every social structure produces a system of thought related to death, outlining questions and creating answers, but in spite of this, the topic remains full of a "cultural unpreparedness". This unpreparedness, as emerged from the research, denotes the paradox of finding oneself powerless in the face of a topic on which one should theoretically be extremely well prepared. Not only is death the only existential certainty common to every human being, it is also an event that pushes every society to produce multiple cultural tools to conceptualize, understand and accept it. In essence, it cannot be said that it is neither a surprise nor that we do not have the ideological tools to deal with it, yet in the contemporary world it seems to be victim of a damnatio memoriae.

This also affects communication with loved ones when they are faced with the possibility of their death, making this event even more difficult to deal with. This is evident in an interesting anecdote told me by B regarding a common situation that happen inside a hospice:

B: “...this situation is created, which is very frequent at the hospice. Wife and husband, the husband is hospitalized, the wife goes to the ward [...] she goes to the head nurse and says "look, I know that my husband has a tumor and that he is at the end of his life, but don't tell him, he doesn't know, we don't tell him, we say that he is here now, he is undergoing treatment and so on". The wife goes away, the husband calls the head nurse and says "look, I know I have a tumor...I have something bad and 
I won't go out of here except with my legs...with my feet forward, but don't tell my wife because she won't stand it".

There is, in those cases, a great attention on the health of the body but zero attention on the health of the relationships. Wouldn't it be better if wife and husband, both aware of the inevitable death of one or the other, could talk freely about it, communicate their sadness instead of keeping it inside, face the event together and enjoy the last moments? There is a tendency to think that thinking more about death means increasing the sadness of a society, but I believe that in the medical field greater awareness and preparation would make it more serene.

During the research I noticed that an interesting topic returned several times: every different way of ending life is inserted in a different moral landscape more or less accepted. By this I mean that when we spoke more explicitly about euthanasia the moral judgement changed according to whether we were talking about active or passive euthanasia. It can be said that voluntary death is more easily placed within a shared moral scenario when it serves as a palliative of human physical suffering. This means that it is not considered always wrong when a doctor "kills" someone because if that person is suffering is it morally correct to help him/her in any way, also if the therapy will end in death.

This information allow us to reflect on the relation between what is structurally legal and what is individually done: if something is illegal doesn't means that everyone believe that its morally wrong.

In this regard, it is interesting to highlight the fact that many doctors have confirmed to me that, despite being illegal, euthanasia already takes place in Italy:

G: The reason for a law, in my opinion and in the opinion of many physicians as well, is to give legality to something that now might risk happening in the shadows, because it still...happens.

In specific situations, if the patient suffers a lot can talk with the physician to obtain a sedation that, basically, allow him/her to die. This fact exalts two important aspects of the current medical situation in Italy: the best cure for a person does not necessarily mean continuing with the treatment; despite the fact that is illegal, passive euthanasia is considered morally right in specific cases.

Doctors, especially those who work in the intensive care unit, may find themselves in the situation where a patient who has attempted suicide shows up and in that case you have to figure out very quickly how to proceed:

$\mathrm{C}:[\ldots]$ we call the psychiatrist who tries to understand what was the root that led him to this gesture and it happens often, it happens to us very often in all ways: of defenestrated people who do not succeed ... substance abuse or voluntary intoxication, it happened to me even to someone who had an accident and then also put at risk other people and not only themselves ... but we do not address this problem here, we ... only if the patient becomes very very serious and has phases where ... maybe he comes out of the acute phase and then returns, these famous relapses, in the decision of an eventual desistance, in the sense when it is no longer reasonable to go on with the course of treatment, you take into account the fact that that person has been in that condition for that reason, but it is a consideration of desistance, which occurs in certain circumstances, as soon as he arrived ... that is, you transfuse him, you do everything, you operate urgently ... it is not that you do not treat him. 
Basically, it has emerged that the end of life needs to be regulated so as to give a scheme to something which, however, is already being done in the shadows, but when it comes to this regulation, multiple factors of both cultural and moral nature come into play.

The difference between the general legal structure of a society and the numerous specific contexts in which is applied, plus the functional existence of statuses and roles create the phenomenon of differentiation that I call moral-structural schismogenesis.

The word schismogenesis was created by Gregory Bateson almost a century ago (Bateson, 1988) but it is a term full of theoretical potential. If for Bateson the conflict is obviously external, I wanted to incorporate it within the individuals, an incorporation that inevitably brings with it a part of the external social structure. To put it simply, by moral schismogenesis I mean the process that leads to the emergence of an ethical difference within the individual based on status and external roles. My idea is based on the assumption that some statuses and roles are more morally imbued than others on the basis of the actual task they have to perform within society. This causes some roles to develop a kind of autonomous morality independent of the individual who then fits into them, as if the role of "doctor" alone possesses an ethics of its own based on what society expects of those who embody this category. All this means that when a person who already has his or her ideological, cultural and moral background finds himself or herself playing a role that has its own ethics, the moral of the role and that of the individual can come into conflict, thus generating even opposing opinions on the most sensitive issues.

In summary, the analysis of physicians' opinions has brought out some important aspects: the absence of a cultural (as far as the population is concerned) and practical (as far as the medical class is concerned) "preparation" regarding the phenomenon of death with relative isolation of the patient and rupture of his relationship with the doctor; the presence in Italy of a different conception between active and passive euthanasia, exalting how much the phenomenon of voluntary death is accepted more only if it is considered a palliative to an incurable suffering; not everything that is illegal is considered morally wrong, demonstrated by the fact that in certain cases euthanasia is still practiced in Italy; finally, before arriving at a national law, a real education to death is desired.

These opinions have allowed to exalt how much in a legally gray situation the doctor creates a personal moral split, divided between his being both citizen and physicians. The problems that emerged allowed to give more depth to a topic that generally divides opinion into two contrasting sides, those for and those against. The reality of the facts is that there are many more nuances, and the national law must be the result of a much broader educational-cultural process.

\section{Conclusions}

The whole analysis highlights how many problems come into play when we start talking about voluntary death: it is not enough to be for or against this phenomenon to understand its infinite nuances, we must first of all give it the right political and personal, practical and moral weight, so as to understand how much more complex the situation is than we might think.

Often, when we talk about a topic that is so ideologically and ethically connoted, we tend to lose sight of the overall picture, we crystallize on our own positions and criticize those against. Before getting stuck inside one's own idea one should analyze the whole phenomenon so as to understand its various implications in order to take a real position. As far as voluntary death is concerned, this research should make it clear that the same personal opinion can be split, that's why is still complicated to create a law. The main point is that before the law we need a specific cultural preparation, a sort of death education, otherwise the law alone will not have efficacy. 
In conclusion, why should we study this topic? While at a scientific level it allows us to highlight certain aspects of the structure of a society and its relationship with the various individual agencies, at a human level it allows us to improve a situation that still today has no resolution. The controversial status that voluntary death has in many countries of the world is a symptom of a situation that must be changed and, as social scientists, we must study and understand in order to change and improve. The euthanasia issue is part of the research-action strand, and since the discipline in question is medical anthropology, it is necessary to focus here on improving the human health condition in its broadest sense.

While Fassin spoke of Ethics of Life, the study of euthanasia allows us to reflect on the Ethics of Death as a study of the ways in which various social subjects give moral meaning to death through their practices. The end-of-life, being such a symbolically dense topic, tends to present itself as an event imbued with a single moral (or similar morals), and therefore it is thought that it is possible to create a homologated and homologating norm. The difficulty in creating a single law is instead the proof that there are different ethics that are not easy to reconcile, just think of the fact that capital punishment is the institutionalization of a death that is however accepted, this means that the same event (death) is connoted differently. The contrast between death penalty and euthanasia, from a legal and social point of view, allows us to reflect, to use Fassin's words again, not so much on which economies of life are implemented but which economies of death are preferred.

During the first Italian medical anthropology conference (2013) Fassin argued that this discipline should have three characteristics: theory, criticism and politics (Fassin, 2014). As social scientists we have to study theories with a critical approach with the ultimate aim of having a political impact on reality in order to improve people's lives.

Studying the end-of-life is therefore important because it allows us to lean towards understanding and change. There is still a lot that needs to be done in order to achieve a regularization of euthanasia; studying it will one day allow us to reach a situation that will make everyone satisfied.

\section{References}

Andorno, R. (2005), The Oviedo Convention: A European Legal Framework at the Intersection of Human Rights and Health Law, in Journal of International Biotechnology Law, vol. 2.

Bateson, G. (1988), Naven, Einaudi, Torino.

Bauman Z. (1995), Il teatro dell'immortalità. Mortalità, immortalità e altre strategie di vita, Il Mulino.

Beauchamp, T.L.; Childress, F.J. (1979), Principles of Biomedical Ethics, Oxford University Press.

Becker, E. (1973), The Denial of Death, The Free Press, New York.

Clark, D. (2002), Between hope and acceptance: the medicalization of dying, in "The BMJ", n.324.

Clarke, A.E., Mamo, L., Fishman, J.R., Janet, K., S., Ruth Fosket, J. (2003), Biomedicalization: technoscientific transformation of health, illness, and U.S. biomedicine, in American Sociological Review Vol. 68, pp. 161-194.

Eisenberg, L. (1977), Disease and Illness. Distinction between professional and popular ideas of sickness, in Culture, Medicine and Psychiatry vol.1, pp. 9-23.

Elias N. (2011), La solitudine del morente, Il Mulino. 
Fassin, D. (2014), Cinque tesi per un'antropologia medica critica, in AM. Rivista della società italiana di antropologia medica, N. 37, pp. 33-50.

Feifel, H. (1965), The Meaning of Death, McGraw-Hill.

Glover, J. (1990), Causing Death and Saving Life, Penguin, London.

Hayry, H. (1997), Bioethics and political ideology: the case of active voluntary euthanasia, Bioethics n.11.

Jones, D.A., Gastmans, C., Mackellar, C. (2017), Euthanasia and Assisted Suicide, Cambridge University Press.

Kaufman, S.R., Morgan, L.M. (2005), The Anthropology of the Beginnings and Ends of Life, in "Annual Review of Anthropology", n. 34, pp. 317-341.

Kleinman, A. (1980), Patients and healers in the context of culture: an exploration of the borderland between anthropology, medicine, and psychiatry, Berkeley: University of California Press.

Kleinman, A. (2006), What Really Matters. Living a Moral Life Amidst Uncertainty and Danger, Oxford University Press.

Kuuppelomaki, M. (2000), Attitudes of cancer patients, their family member and health professionals toward active euthanasia, European Journal of Cancer Care n. 9, pp. 16-21.

Lewins, F. (1998), The development of bioethics and the issue of euthanasia: regulating, deregulating or re-regulating? Journal of Sociology n.34.

Mauss, M. (2002), Les techniques du corps, electronic edition created by Jean-Marie Tremblay (professeur de sociologie au Cégep de Chicoutimi).

McDougall, J.F., Gorman, M. (2008), Euthanasia, ABS-CLIO, Santa Barbara, California.

Miles, S.H. (2004), The Hippocratic Oath and the Ethics of Medicine, Oxford University Press.

Oduncu, F.S. (2003), Euthanasia: Killing as Due Care? University of Munich, Germany.

Owen, C.; Tennant, C.; Levi, J.; Jones, M. (1992), Suicide and Euthanasia: patient attitudes in the context of cancer, Psycho-Oncology n.1, pp.79-88.

Quaranta I., (edited) (2006), Antropologia medica. I testi fondamentali, Raffaello Cortina.

Preston, S.H. (1976), Mortality Patterns in National Population, Academic Press Inc., New York.

Rosenfeld, B. (2004), Assisted Suicide and the Right to Die, American Psychological Association.

Seale, C. (2000), Changing patterns of death and dying, in "Social Science \& Medicine", n.51, pp. $917-930$.

Singer, P. (1995), Rethinking Life and Death, St. Martin's Press, New York.

Veena, D., Clara, H. (2016), Living and Dying in the Contemporary World, University of California Press.

Yolanda, M., G. (2018), Euthanasia and Ethical Dilemmas at the End of Life, in Wulfenia Journal, vol.25. 